in-depth study of its internal mechanism is of great significance for the prevention and treatment of gout.

Objectives: This paper mainly discussed the expression of peripheral blood immune function in patients with gouty arthritis and the changes and significance of peripheral blood immune function in gout with different uric acid levels.

Methods: A retrospective analysis was performed on 258 outpatients and inpatients with gout in shanxi medical university from 2016 to 2019, all of which met the diagnostic criteria of the American college of rheumatology (ACR) in 1997, and 41 healthy controls. Complete clinical data and general laboratory data were collected, and peripheral blood lymphocyte and CD4+T cell counts were completed for all subjects.

Results: (1) Total peripheral blood B cells of gout patients [238.00 $(171.50,323.07)$ and $191.04(149.66,253.14), Z=-2.759, P=0.006]$, Th cells [814.11 $(617.50,1052.89)$ and $625.84(562.52,750.15), Z=-3.905, P<0.001], T h /$ Ts $[1.4(1.04,2.00)$ and $1.11(0.89,1.52), Z=-2.862$, Th17/Treg $[0.36(0.20,0.60)$ and $0.24(0.14,0.34), Z=-3.949, P=0.000]$ and the absolute counts of Th17 cells [9.06 $(5.07,15.57)$ and $7.48(4.31,10.18), Z=-2.520, P=0.012]$ were higher than those of the healthy control group, and the differences were statistically significant. The absolute count of Treg cells [28.82 (17.48,38.04) and $30.22(22.74,39.46)$, $\mathrm{Z}=-2.249, \mathrm{P}=0.025]$ was lower than that of the healthy control group, and the difference was statistically significant. (2) The Th17\% [1.05 $(0.71,1.42)$ and 1.27 $(0.73,2.00), Z=-1.995, P=0.046]$ and the Th17/Treg $[0.25(0.14,0.44)$ and 0.39 $(0.23,0.63), Z=-3.147, P=0.002$ ] in peripheral blood of patients with high uric acid in the gout group were higher than those in the normal uric acid group, the difference was statistically significant. The Treg \% [3.84 $(2.65,5.02)$ and 3.12 (2.36,4.37), $Z=-2.239, P=0.025]$, and the Treg cells $[30.75(21.97,43.27)$ and $24.07(16.84,36.29), Z=-2.522, P=0.012]$ were lower than those in the uric acid control group, with statistically significant differences.

Conclusion: The level of Th17 cells in peripheral blood of patients with gout increased significantly while the level of regulatory $T$ cells decreased significantly. Th17 cell level in peripheral blood of the high uric acid group was significantly increased compared with the normal uric acid group, while the regulatory $T$ cell level was also significantly decreased, and the Th17/Treg ratio was also increased. This suggests that regulatory $T$ cells may play an important role in the pathogenesis of gout and are closely related to uric acid metabolism, so the study of internal mechanism can provide a new target for the treatment of gout.

References:

[1] Ragab G, Elshahaly M, et al. Gout: An old disease in new perspective - A review[J]. Adv Res. 2017;8(5):495-511. DOI: 10.1016/j.jare.2017.04.008.

[2] Dai XJ, Tao JH, et al. Changes of Treg/Th17 Ratio in Spleen of Acute Gouty Arthritis Rat Induced by MSU Crystals. [J]. Inflammation. 2018;41(5):19551964. Doi: 10.1007/s10753-018-0839-y.

Disclosure of Interests: None declared

DOI: 10.1136/annrheumdis-2020-eular.1594

\section{Infection-related rheumatic diseases}

\section{AB0937 COMPARATIVE STUDY OF PYOGENIC SPONDYLODISCITIS VERSUS TUBERCULOUS SPONDYLODISCITIS}

A. Ben Tekaya ${ }^{1}$, L. Ben Ammar², M. Ben Hammamia ${ }^{3}$, O. Saidane ${ }^{1}$, S. Bouden ${ }^{1}$, R. Tekaya ${ }^{1}$, I. Mahmoud ${ }^{1}$, L. Abdelmoula ${ }^{1} .{ }^{1}$ Charles Nicolle Hospital,

Rheumatology, Tunis, Tunisia; ${ }^{2}$ Kassab Institute, Rheumatology, Tunis, Tunisia; ${ }^{3} \mathrm{La}$ Rabta Hospital, Cardiovascular Surgery, Tunis, Tunisia

Background: Infectious spondylodiscitis is a life-threatening infection of the intervertebral disc and adjacent vertebrae that can be caused by a variety of microorganisms. Our country is at intermediate endemicity for Tuberculosis.

Objectives: To compare the clinical, biological, radiological characteristics, management and prognosis of pyogenic versus tuberculous spondylodiscitis.

Methods: Retrospective study of 89 patients admitted to our department over a period of 20 years [1998-2018]. The diagnosis of spondylodiscitis was made based on clinical, biological, radiological and bacteriological data.

Results: The average age of the patients was 56.1 years. There were 46 men $(51.6 \%)$ and 43 women $(48.4 \%)$ with a female predominance during tuberculous spondylodiscitis $(57.1 \%)$ versus $26.9 \%$ during pyogenic spondylodiscitis ( $p$ $=0.009$ ).

Diabetes was more frequent during pyogenic spondylodiscitis but with no statistically significant difference $(p=0.4)$. The evolution time was statistically greater during tuberculous spondylodiscitis $(p<0.001)$. Patients with tuberculous spondylodiscitis had more frequently an impaired general condition $(p=0.02)$.

Hyperleukocytosis was noted more frequently in the pyogenic group than in the tuberculosis group $(p=0.03)$, while the increase in sedimentation rate was not significantly different between the two groups $(90 \mathrm{~mm} / \mathrm{h}$ and $76 \mathrm{~mm} / \mathrm{h}$, respectively, $p=0.1$ ). We found no statistically significant difference regarding the site of spondylodiscitis.

Radiologically, the frequency of para-vertebral and psoas abscesses, epiduritis and the presence of spinal cord compression were similar in the two groups $(p=0.2 ; p=0.1$ and $p=0.1$, respectively), whereas mirrored geodes were more frequent during tuberculosis $(p=0.04)$.

Surgical and interventional treatments (percutaneous sampling, abscess drainage) were more frequently noted during pyogenic spondylodiscitis, but without significant difference $(p=0.2)$. The occurrence of immediate complications was more frequent during tuberculosis but without a statistically significant difference $(\mathrm{p}=0.2)$.

Conclusion: In our series, patients with tuberculous spondylodiscitis tend to have a chronic pattern of progression and more often an impaired general condition. However, there was no significant difference in the presence of abscess, epiduritis and the occurrence of complications between tuberculous spondylodiscitis and pyogenic spondylodiscitis.

Disclosure of Interests: None declared

DOI: 10.1136/annrheumdis-2020-eular.4834

\section{AB0938 PARTICULARITIES OF INFECTIOUS SPONDYLODISCITIS INTHE ELDERLY: COMPARATIVE STUDY WITH THE YOUNG SUBJECT}

L. Ben Ammar ${ }^{1}$, A. Ben Tekaya ${ }^{2}$, M. Ben Hammamia ${ }^{3}$, O. Saidane², S. Bouden², R. Tekaya ${ }^{2}$, I. Mahmoud ${ }^{2}$, L. Abdelmoula ${ }^{2}{ }^{1}{ }^{1}$ Kassab Institute, Rheumatology, Tunis, Tunisia; ${ }^{2}$ Charles Nicolle Hospital, Rheumatology, Tunis, Tunisia; ${ }^{3}$ La Rabta Hospital, Cardiovascular Surgery, Tunis, Tunisia

Background: The incidence of infectious spondylodiscitis is steadily increasing. The prognosis depends on various parameters, including age.

Objectives: To explore the differences in presentation and the results of further investigations and the prognosis of spondylodiscitis between young and elderly subjects.

Methods: This is a retrospective study of 113 patients admitted to our department over a period of 20 years [1998-2018]. The epidemiological, clinical, biological, radiological and progressive aspects of spondylodiscitis in subjects over the age of 65 were compared to cases of spondylodiscitis in young subjects whose age is less than 65 years.

Results: Of the 113 identified patients, $33(29.2 \%)$ were classified as older, with male predominance in both groups ( 56.2 and $51.5 \%$ respectively; $p=0.6$ ). The presence of risk factors, particularly diabetes, was more frequent in the older subjects but without a statistically significant difference $(p=0.2)$. Hepathopathies were also more frequent in the elderly but without statistically significant difference $(p=0.3)$. The mean duration of progression was 3 months with no statistically significant difference between the two groups ( 4 and 5 months respectively, $p=0.1$ ). The presence of neurological symptoms, especially radiculalgia, was more frequently reported in younger subjects $(p=0.01)$. Also, young subjects were more likely to have a fever $(p=0.005)$. On the other hand, a biological inflammatory syndrome was more frequently encountered in older subjects $(p=0.03)$.

We found no statistically significant difference in the location of spondylodiscitis $(p=0.4)$. Also, multifocal involvement was more frequent in older subjects but without a statistically significant difference.

Radiologically, para-vertebral abscesses, epiduritis and spinal cord compression were more common in the elderly group $(p=0.03 ; p=0.01$ and $p=0.01$, respectively). While the frequency of intervertebral pinching and erosions was similar in both groups ( $p=0.1 ; p=0.8$, respectively).

Tuberculosis was the most involved germ in more than $50 \%$ of cases with no statistically significant difference between the two groups (57.5 and $51.5 \%$ respectively; $p=0.5$ )

All patients received targeted antibiotic therapy.

The occurrence of immediate complications was more frequent in the elderly but without a statistically significant difference $(p=0.1)$. They included neurological complications (spinal cord compression and Cauda equina syndrome) that occurred in $6.3 \%$ of cases in younger subjects versus $36.4 \%$ of cases in the elderly $(p=0.07)$. Followed by treatment side effects dominated by disturbance of liver function due mainly to antituberculosis drugs occurring in $16.3 \%$ of cases in the younger group and $15.2 \%$ of cases in older subjects $(p=0.8)$. Finally, there were 2 deaths in each group $(\mathrm{p}=0.5)$.

Conclusion: Our series has shown that the picture of spondylodiscitis in the elderly is less noisy. However, the neurological damage is more pronounced on imaging. The misleading symptomatology in the elderly explains the delay in diagnosis and treatment, which leads to more frequent complications and excess mortality.

Disclosure of Interests: None declared

DOI: 10.1136/annrheumdis-2020-eular.4855 